\title{
UN CURSO DE ACTUALIZACIÓN Y CAMBIOS CONCEPTUALES EN PROFESORES DE FÍSICA
}

\author{
PACCA, J. L., PACCA, A. y VILlani, A. \\ Instituto de Física. Universidad de São Paulo. Caixa Postal 66318 05389-970. São Paulo. SP, Brasil.
}

\section{SUMMARY}

The behaviour of secondary school Physics teachers at an updating course was analysed; the results led to an indication of a conceptual change in their practice. The object of analysis and discussion was the actual pedagogical planning by the teacher. The dynamics developed in the course favoured the reelaboration of the teacher's conceptions, not only in relation to Physics contents but also in relation to the students' learning process; a conceptual change in three sequent phases could be characterized.

\section{INTRODUCCIÓN}

Poner al día los profesores de ciencia es un tarea que se está haciendo cada vez más importante debido a las crecientes dificultades que los profesores encuentran en su día a día en las clases y al progreso científico de las ciencias exactas y de la educación.

Los programas de actualización con el objetivo de promover cambios sobre cómo enseñar ciencias se enfrentan a dos desafíos: el primero supone modificar las concepciones científicas del propio profesor y el segundo modificar sus ideas y práctica de enseñanza. Estos dos ejes se pueden reforzar mutuamente contribuyendo a tomar conciencia sobre el papel del profesor en el desempeño de sus funciones como orientador del proceso de aprendizaje, así como de las estrategias a utilizar capaces de vencer las dificultades de los estudiantes. Una de las metas más importantes del área de educacion científica es la de realizar investigaciones y encontrar resultados que contribuyan a la toma de conciencia.

Las dificultades en la interiorización del contenido son graves en los países de América Latina debido al problema crónico de escasa formación de los profesores durante el período de formación. Por ejemplo, los profesores de un curso de actualización en Colombia fueron incapaces, incluso después de mucha discusión en grupo, de percibir las contradicciones entre los argumentos utili- zados por ellos y las leyes de Newton al resolver problemas simples de mecánica (Zalamea y Paris, 1989). El estudio de casos realizado por Barnes y Barnes (1989) mostró que, a pesar de la atención personalizada y específica, no todos los profesores consiguieron alcanzar un aumento significativo en la comprensión deI contenido que se enseñaba.

Fueron también objeto de investigación la influencia que el dominio de la disciplina por parte del profesor tiene en la elección de las actividades escolares y en la forma en que éste guía sus clases (Carlsen, 1991), así como la barrera constituida por la falta de conocimientos científicos que dificulta su participación en actividades innovadoras (Tobin y Espinet, 1989).

En el trabajo de Hewson (Hewson y Hewson, 1987) se exponen en parte las resistencias para modificar la concepción de «enseñanza de las ciencias». A partir de él se puede concluir que incluso un programa bien presentado y con discusión de nuevas maneras de enseñar no se traduce inmediatamente en una mejora de las actividades escolares correspondientes. Otros estudios de casos más profundos (Briscoe, 1991) muestran la influencia de creencias, imágenes y modos de ver del profesor en su práctica docente, pudiendo inclusive constituir bloqueos inconscientes para la práctica de nuevas actividades docentes. 
A pesar de las sugestiones para modificar las actitudes y prácticas de los profesores (McDermott, 1990, Gil y Pessoa, 1992), las informaciones sobre el proceso de cambio conceptual del profesor a lo largo de un curso de actualización son aún escasas; sin embargo, parecen ser necesarias para mejorar los cursos haciéndolos compatibles con las posibilidades y las expectativas del profesorado (Villani y Pacca 1992).

En una investigación desarrollada en el Instituto de Física de la USP (Universidad de S. Paulo, Brasil) se investigó la actuación de nueve profesores de física de segundo grado' en un programa de actualización. Éste tuvo como objetivo modificar la forma de enseñar en clase y desarrollar material didáctico organizado para promover el aprendizaje de la mecánica (Pacca y Villani, 1992). La duración del curso fue aproximadamente de 200 horas. Fue adoptada la planificación pedagógica como el objeto de trabajo concreto para desarrollar el programa. Consideramos que éste es un instrumento práctico de control y facilitador del aprendizaje (Villani, 1991; Pacca, 1992).

Los datos se obtuvieron mediante grabaciones sonoras de entrevistas e interacciones en grupos reducidos, observaciones sistemáticas de las interacciones entre los participantes y del análisis de los trabajos escritos producidos durante las sesiones o en casa (Bodiao, 1993). EI análisis y observación de la actuación de los profesores se centraron en la elaboración y discusión de la planificación de una unidad didáctica sobre la ley de inercia y su aplicacion en clase, aunque durante las 200 horas de curso hayan sido estudiadas las tres leyes.

Fueron apareciendo progresivamente las dificultades encontradas por los profesores en la elaboración de una planificación para la enseñanza de la ley de inercia. Eran abordadas en la medida de su propia concienciación de los problemas y de su posible aplicación en clase. Hemos encontrado fases de desarrollo bastante diferenciadas en la elaboración de los programas de los profesores (Pacca y Villani, 1991). En este trabajo procuraremos caracterizar estas fases a través de los cambios de perspectivas de Ios profesores y de la relación con la mejora de sus concepciones científicas y de sus competencias didácticas.

\section{CONDICIONES INICIALES DE LOS PROFESORES}

La comprensión de la ley de inercia entre estudiantes e incluso profesores de segundo grado no es común y parece obedecer a un proceso gradual (Viltani y Pacca, 1991). Esta ley es básica dentro de la teoría newtoniana, yá que constituye el criterio para distinguir los sistemas referenciales inerciales.

Al inicio del curso de actualización, los conocimientos de los profesores presentaban una diferenciación considerable, pudiendo ser caracterizados por diferentes niveles de comprensión. Por el contrario, las planifícacio- nes que realizaban parecian obedecer a esquemas generales semejantes, aunque con alguna diferencia poco significativa desde el punto de vista de control del aprendizaje.

En cuanto a los conocimientos relacionados con la ley de inercia, la comprensión de algunos profesores no pasaba del nivel fenomenológico. Éste se resume en la idea de que, en ausencia de fuerzas de rozamiento, los cuerpos continúan en reposo o en movimiento rectilíneo uniforme. El resultado encontrado para un problema constituía en general una información final y seguían apareciends) varios tipos de ideas espontáneas en relación con fuerza y movimiento, movimiento y sistema de referencia, y entre movimientos en direcciones diferentes, sin que se percibiera ninguna contradicción con la visión de la física expresada por la ley de inercia. Sin la posibilidad de una articulación más conectada con otras informaciones, el conocimiento adquirido parecía tener una vida breve y no adquiría características deductivas, típicas de una explicación estructurada y general.

Fn estas condiciones, realizar la tarea de elaborar la planificación de la ley de inercia se hacía doblemente frustrante: parecía contrariar las expectativas generalizadas de los profesores de aprender informaciones útiles para ser aplicadas directamente en sus clases, al mismo tiempo que se reducía al trabajo rutinario de elaborar una lista de tópicos con una secuencia muy semejante a la del libro de texto adoptado. Los objetivos sugeridos en la planificación eran extremadamente genéricos $y$, consecuentemente, las evaluaciones propuestas se alejaban de la tentativa de apreciar la comprensión de puntos específicos esenciales. Además, la falta de dominio del contenido parecía inhibir la sugestión de actividades más interactivas y productoras de discusiones interesantes en clase.

Otros profesores podian ciasificarse en un segundo nivel de comprensión que fue llamado cuasiformal. Bstos asimilaron buena parte de las características fundamentales de la ley de inercia. De esta forma, la ley de inercia era contemplada más como un conjunto de propiedades relacionadas con el comportamiento de las magnitudes que intervienen en el movimiento que como un principio capaz de definir los sistemas de referencias inerciales de la mecánica y conectarse con otros principios como el de acción y reacción. De forma general, el aprendizaje ya habia alcanzado una relativa estabilidad y el contenido aprendido podía, eventualmente, ser el centro de referencia de nuevas informaciones a pesar de que en algunas circunstancias aparecieron algunas concepciones espontáneas.

La planificación inicial de los profesores de este nivel de comprensión era en cierta forma más variada que el anterior. La lista de tópicos para ser abordados obedecía a criterios más personales y la lista de ejercicios para ser discutidos estaba más elaborada. Aparecían también actividades más abiertas como el uso del laboratorio para probar la ley; por ejemplo, con una experiencia sobre un movimiento en un plano con reducción gradual del rozamiento (PSSC, 1960). Sin embargo, los objeti- 
vos a alcanzar se mantenían distantes de la práctica efectiva: no focalizaban los conceptos más importantes a aprender, no se referían a las eventuales etapas en la comprensión o habilidades de los estudiantes. Finalmente, Ias evaluaciones eran variaciones más o menos originales de los ejercicios propuestos sin una relación estrecha con los objetivos ni con las concepciones alternativas de los estudiantes.

A pesar de ser más personales, las unidades didacticas realizadas por los profesores de este nivel de comprensión tenían una característica común: focalizaban exclusivamente el contenido científico a ser enseñado siguiendo una secuencia lógica para el profesor y no presentaban indicios de preocupación con las concepciones de los estudiantes ni con las dificultades o barreras que podrían representar. Por ello no proponían esquemas de seguimiento más sistemático del progreso ni de las formas de pensar sobre el asunto por parte de los estudiantes. Las actividades interactivas y prácticas tenían poco espacio para la điscusión y exposición de dudas.

La comprensión de la ley de inercia para la mayoría de Ios profesores no pasaba de estos dos niveles; sólo el profesor A mostró un dominio del asunto a un nivel formal. Para él, la ley de inercia era un principio general a partir del cual podían deducirse consecuencias y debía ser coordinado con las restantes leyes de Newton en la solución de los problemas de mecánica. Todos los recursos de razonamiento eran utilizados en la solución de los problemas junto con la rápida percepción de sus eventuales fallos. Prácticamente no existieron barreras cognitivas y sólo aparecieron dificultades esporádicas ał abordar tareas más complejas. La planificación inicial de este profesor fue interesante y reveló la preocupación por una comprensión más profunda de la ley đe inercia que procuró desarrollar mediante una serie bastante grande de cuestiones de discusión. Algunas presentaban retaciones con las ideas no científicas detectadas en la investigación previa pero no se le daba una relevancia especial. Para este profesor la discusion en clase, dirigida por él, conseguiría tarde o temprano llevar a la mayoría de los estudiantes a un conocimiento adecuado. Por este motivo afirmaba que no estaba preocupado con la evaluación, considerándola una actividad poco creativa; prefería fundamentalmente las discusiones en pequeños grupos o de toda la clase. De hecho, se puede considerar que dentro de la dinámica de clase ya hacía las evaluaciones y redirigia las cuestiones. Sus objetivos explícitos eran, sin embargo, generales y se referían al desarrollo cultural y a la formación humana de sus atumnos.

\section{ALCANZANDO UN CONOCIMIENTO CIENTIFICO MÁS SATISFACTORIO}

El curso tenía como objetivo explícito la elaboración de una unidad didáctica personal, coherente con la capaci- dad del profesor y capaz de controlar el aprendizaje de los alumnos.

Las primeras actividades fueron discusiones de problemas típicos extraidos de las investigaciones sobre concepciones alternativas seguidas de tareas de aplicación a sus propios alumnos y recogida de información. Se podía pensar que el análisis de las respuestas de los estudiantes constituía una fuente interesante de información para los profesores y que éstas desencadenaban la búsqueda de actividades capaces de modificar las concepciones de los estudiantes. Por el contrario, la preocupación común de los profesores, independientemente de su nivel de comprensión, se dirigió hacia el perfeccionamiento de su propio conocimiento científico.

Los que estaban en el nivel fenomenológico percibieron la fragilidad de sus conocimientos; no fueron capaces de distinguir entre nociones cientificas y no científicas. Para ellos resultaba muy diff́cil la resolución de problemas, incluso simples, como la elaboración de un gráfico del movimiento de un cuerpo en un campo de fuerza constante. Un caso interesante sucedió con el profesor $\mathbf{F}$, que después de las discusiones sobre movimiento relativo, afirmaba con convicción que las velocidades y las trayectorias dependían del sistema de referencia, pero insistía en colocar una fuerza en la dirección del movimiento cuando necesitaba explicar su velocidad.

La discusión de la planificación de las unidades didácticas de los compañeros parecía estimular la necesidad de mejorar los propios conocimientos. Algunos profesores intentaron aclarar el problema introduciendo la lectura de textos sobre las experiencias y conclusiones de Galileo relacionadas con la inercia. Un poco más tarde se percataron de que éstos no aclaraban el problema ni para los alumnos ni para ellos mismos.

El nivel de comprensión fenomenológico permite elaborar una planificación estrechamente relacionada con e] contenido científico ya estructurado y acabado y con la secuencia lógica que lo describe. Sin embargo, no permite una visión general ni la posibilidad de operar deductivamente para resolver problemas nuevos. En la planificación de los profesores de esta fase parecía que faltaba coordinar los experimentos sobre el movimiento en el plano (que estudia Ias fuerzas de rozamiento como causa de la disminución de la velocidad) y el cambio de sistema de referencia (que hace físicamente equivalente el reposo y el movimiento rectilíneo uniforme).

Una actividad bastante importante en esta fase que se fue imponiendo en sesiones sucesiva fue la resolución y discusión de problemas que eran expuestos por los profesores como ejemplos de recursos didácticos y que ellos mismos utilizaban con sus alumnos. Fueron precisas muchas horas de trabajo y discusión para comprender el contenido de los problemas con la exactitud y rigor necesarios. Se procuró tratar las cuestiones teniendo en cuenta las relaciones matemáticas adecuadas para resolverlas. Siempre que fue posible se siguieron las pistas y razonamientos ofrecidos por los profesores en la obtención de soluciones. 
Paralelamente a estas actividades, el profesor tenía como trabajo de casa probar su unidad didáctica con los alumnos. Así, los profesores, al mismo tiempo que mejoraban su nivel de comprensión, modificaban su unidad haciéndola más incisiva a través de ejemplos y actividades que apuntaban objetivamente hacia el contenido. Es decir, la búsqueda de otras actividades que implicasen más a los estudiantes corría paralelo con su empeño en alcanzar mayor precisión y rigor de contenido. Como dice el profesor $F$, refiriéndose al problema de la bomba dejada caer desde un avión:

«Resalté los desplazamientos horizontales de la bomba, porque ésta había sido también mi duda; me di cuenta de que debía ser eso. Parece que la seguridad en el contenido y haber vivido las dificultades lieva al profesor a ser más ágil.»

De hecho, la aplicación de la unidad didáctica en la realidad escolar se mostró fundamental para la creación de una nueva concepción de la enseñanza. La disposición del profesor para encontrar explicación al fracaso de sus alumnos y descubrir lo que ocurre en términos de dificultad conceptual le lleva a meditar sobre el contenido y a reelaborar sus propias ideas acerca del proceso enseñanza-aprendizaje.

Los profesores que se encontraban en un nivel más elevado de dominio del contenido también dedicaron sus esfuerzos al perfeccionamiento del conocimiento científico. Este nivel de comprensión no garantizaba aún la transferencia plena de las informaciones adquiridas en las discusiones durante el curso para el medio escolar. El profesor no siempre conseguía descubrir, en las respuestas de los estudiantes, concepciones incoherentes con las ideas científicas. Por ejemplo, el profesor B hizo una evaluación de sus alumnos concluyendo que habían aprendido el principio "con la excepción de cierta confusión que aparecía con respuestas dadas posteriormente». Esta «confusión" nos indicaba que las concepciones no cientificas estaban aún presentes y que debían ser modificadas.

El mismo profesor anterior, después de analizar experimentalmente con sus alumnos el movimiento parabólico de una bola lanzada horizontalmente, consideró resuelto el problema de la composición de movimientos en direcciones diferentes. Más tarde se percató de que los estudiantes fallaban en problemas físicamente iguales pero con características peculiares que hacían aflorar concepciones no científicas. Por ejemplo, los alumnos no reconocian en una bala de fusil disparada horizontalmente una situación idéntica al experimento estudiado anteriormente en detalle.

Para el profesor no fue fácil ni inmediato ver en las equivocaciones de los alumnos formas de concebir inadecuadas que deberían ser consideradas y discutidas con una orientación objetiva y concreta. Incluso el profesor que tenía inicialmente un conocimiento más estructurado no consiguió durante esta primera fase modificar substancialmente su planificación en el sentido de preocuparse del aprendizaje efectivo y de la reelaboración de las concepciones alternativas de sus alumnos.

En general, en esta primera fase, la planificación aún no se enfrentaba a la concepción del alumno sobre la necesidad de una fuerza siempre que existe un movimiento. Las experiencias, lectura y discusión de textos históricos no parecían modificar esa concepción.

Las discusiones en grupo tuvieron un papel decisivo en la mejora del nivel de conocimiento de todos los profesores y especialmente el cuestionamiento de los compañeros que tenían la tarea de presentar y justificar en los plenarios sus propuestas para la clase. Estas actividades les familiarizaron con las diferentes manifestaciones de las ideas no científicas y les obligaron a organizar las ideas adecuadas desde el punto de vista de la física. Un problema enunciado por los propios profesores se erigió como símbolo de esta actividad:

«Si tuviéramos un coche con velocidad v sobre la superficie de la Tierra y eliminásemos todos los rozamientos éste daría una vuelta completa en torno a la Tierra. ¿Cómo es compatible con la ley de la inercia sabiendo que la resultante de las fuerzas que actúan sobre el coche es nula (el peso y la reacción normal de la Tierra)?»

Las discusiones intensas en torno a las hipótesis (casi siempre alternativas) que podrían resolver el dilema y las dificultades de cuestionar la igualdad entre peso y reacción normal permitían darse cuenta del conjunto de ideas alternativas no científicas así como de la aplicación correcta de la ley de inercia.

\section{ORGANIZANDO ACTIVIDADES PARA IAA CLASE}

La búsqueda de actividades que hiciesen intervenir más a los estudiantes avanzaba junto con el empeño del profesor en conseguir precisión y rigor en el contenido. Las barreras encontradas ał aplicar su planificación en clase favorecían la adquisición de ese conocimiento específico.

Incluso después de adquirir el profesor una comprensión razonable del contenido existía un desfase en la transferencia a la planificación. No era inmediata la incorporacion de las concepciones de los estudiantes a los elementos que forman parte en la elaboración de la planificación. Para resaitar y caracterizar una incoherencia conceptual no científica, como «siempre que hay movimiento existe una fuerza resultante no nula», sería necesario coordinar dos informaciones: velocidad constante sin fuerza actuante y relatividad del movimiento.

Un caso interesante sucedió con el profesor B: para evaluar si sus alumnos habían comprendido el principio después de discutir y resolver problemas como los mencionados, propuso un problema que él crcía semejante a los solucionados en clase. Ell resultado fue, una vez más, un fracaso, Io que mostraba que la comprensión de los 
estudiantes era sólo local, pues no presentaba características deductivas o de transferencia a otros contextos; exactamente las mismas dificultades por las que había pasado el profesor al principio del curso. En este punto, algunos docentes ya eran capaces de identificar con precisión las dificultades de sus alumnos, como muestra el siguiente párrafo:

«La dificultad de los estudiantes en el análisis de la caída de un tornillo en un tren en movimiento uniforme radicaba en aceptar la equivalencia física entre reposo y movimiento.»

Probablemente la relativa seguridad sobre gran parte de Ios puntos críticos del principio de inercia permitió y estimuló a casi todos los profesores a fijar su atención en las concepciones alternativas de los estudiantes. La primera actividad en casi todas las planificaciones fue educir y analizar cualitativamente las respuestas de sus alumnos con cuestionarios específicos. La segunda fue la discusión de ejemplos típicos que debían convencer a los alumnos a adoptar las nuevas ideas y abandonar las antiguas. La actividad de evaluación final estaba formada por uno o más problemas semejantes a los discutidos en clase desde el punto de vista físico.

El profesor E, que al organizar sus clases nunca se había alejado del tipo de clase expositiva, comentaba con satisfacción su capacidad de dirigir una discusión:

«Hice la experiencia de la gota de agua en el tubo de aceite. Los alumnos esperaban que Ia gota mayor cayera más despacio y lo que vieron fue lo contrario.»

Esta modificación en la calidad y estructura de la planificación produjo varios efectos en el desarrollo de las actuaciones de los profesores y de sus prácticas didácticas, al menos por lo que se podía deducir de sus explicaciones.

La perspectiva de alcanzar un objetivo bien definido parecía inyectar ánimo a los profesores incluso en condiciones institucionales adversas, como muestran los siguientes ejemplos:

«Este año los alumnos parecen más interesados que Ios de años anteriores a pesar de las huelgas sucesivas de los profesores» $(\mathrm{E})$.

«En mis clases no daba mucha importancia a las jeyes de Newton; fue del año pasado para éste cuando me fascinó. Es necesario que esté claro lo que quiero exigir de] alumno en clase» (B).

Probablemente el interés de los alumnos era consecuencia uel aumento significativo del interés y disponibilidad del propio profesor en interacción con la clase proponiendo actividades significativas.

La actividad denominada «diario de a bordo» (descripción de los problemas y reflexiones de cada profesor al comienzo de cada sesión del curso de actualización) adquirió cada vez más un papel fundamental en la defi- nición de los asuntos a ser discutidos con un evidente atumento del interés y participación de los profesores. Los participantes parecían haber modificado su percepción sobre ja forma de actuar de sus ałumnos y mucho más rápidamente que antes eran capaces de localizar fallos en el proceso de aprendizaje. Los profesores más sensibles intentaban soluciones más innovadoras ante las dificultades. El profesor D, por ejemplo, comprendió que sus alumnos seguían el razonamiento propuesto (aumento de la duración del estado de movimiento con la disminución de las resistencias) hasta cierto punto, rehusando las últimas consecuencias (movimiento continuo en la ausencia de fuerzas de rozamiento).

«Intenté desplazar el movimiento hacia el espacio distante donde se podría suponer que había ausencia de fuerzas. Parece que esta idea es más aceptable entre los alumnos. Quizás el contraste entre to que experimentan en la vida cotidiana y la situación límite sea demasiado grande para iniciar una reflexión... Oyendo a los alumnos corregí el rumbo y obtuve éxito.»

El mismo profesor, más adclante, intentando abordar el problema de la "fuerza impresa», afirmaba:

«Parece que la concepción de fuerza concebida por los alumnos no es exactamente la misma que la de física, entonces introduje la "energía del movimiento" para suplir la ansiedad que sienten cuando se habla de movimiento sin fuerza.»

En la opinión de este profesor se trataba de una solución provisional hasta que más tarde se introdujera otra magnitud física.

"Sólo se consigue eliminar "la fuerza impresa" cuando se introduce la cantidad de movimiento y energía, porque entonces el alumno tienc otros elementos para referirse a lo que ve.»

El profesor $\mathrm{C}$, intentando resolver el problema de coordinar las ideas que componen la ley de inercia con sus ałumnos, recurrió a la discusión de los textos sobre los experimentos de Galileo (Koyré, 1986). Despućs de reflexionar sobre el contenido a tratar en clase concluyó:

« QQué significa para el alumno que la piedra caiga a los pies del mástil? Hay que mostrar que noes necesario que tenga una fuerza impresa... Tengo que exponer el problema: ¿es posible que exista movimiento sin fuerza? Y Ilevar al alumno a la reflexión... En una discusión en grupo cambian ideas y experiencias y entienden mejor.»

Ese profesor, para ayudar a sus compañeros a comprender mejor la ley de inercia, decidió escribir un trabajo en el que describió el camino intelectual recorrido y las dudas experimentadas por él mismo.

De los nueve profesores que siguieron el curso hasta cste punto sólo el profesor $G$ no consiguió abordar con relativa seguridad las concepciones de los estudiantes. Presentaba mucha dificultad superar el nivel fenomenológico de comprensión a pesar de los esfuerzos of recidos 
para vencer sus deficiencias. Prefería creer que podía conseguir resultados positivos incidiendo en la metodologia de la clase.

\section{EVALUANDO EL APRENDIZAJE Y LA PLANIFICACION}

Lo máximo que algunos profesores $(\mathrm{E}, \mathrm{H}, \mathrm{I})$ consiguieron alcanzar en la segunda mitad del curso fue incluir en la planificación las actividades que facilitaban la discusión de ideas alternativas de los estudiantes. El próximo paso para ellos fue controlar las actividades para hacerlas más adecuadas a stu realidad institucional y social. Para los restantes profesores, el proceso fue más adelante.

A partir de este punto, otros factores identificados que generan problemas a los profesores para desarroIlar su trabajo (falta de condiciones en general o en la forma de espacio y equipos, desinterés de los estudiantes y desmotivación asociada a la indisciplina en clase), perdieron su relevancia o pasaron a ser tratados de un modo objetivo. Por un lado, el profesor se sentía más seguro de su saber, oía a sus alumnos y discutía con ellos las dudas presentadas. Por otro Iado, era capaz de comprender mejor el significado de las dudas, consecuencia de una concepción más amplia que la sustentaba, y además era capaz de establecer los puentes adecuados para transformarlas en los contenidos físicos correctos.

La aplicación de la planificación en clase comenzaba a dar sus resultados. Sin duda, los alumnos mostraban una participación más intensa pero los resultados de las evaluaciones aún no eran aceptables. Las concepciones no científicas eran realmente difíciles de modificar a pesar de que las discusiones en clase mostraban muchas veces que algo había sido aprendido y era utilizado correctamente. En este sentido, el comentario del profesor $\mathrm{C}$ marcó el inicio de un cambio de perspectiva que se concretizó en la atención al aprendizaje efectivo de los estudiantes:

«Hacemos del aprendizaje una cuestión de acierto-error sin importarnos cómo el alumno liegó a la respuesta. Más importante que simplenente acertar es qué fue lo que hizo para llegar a la respuesta -el proceso de aprendizaje.»

Esta situación experimentada por varios profesores se convirtió en un asunto repetitivo en los relatos y discusiones en el «diario de a bordo» Los profesores más adelantados desplazaron progresivamente el foco de reflexión hacia el proceso de aprendizaje, su estabilidad y sus condiciones:

«¿Por qué los alumnos olvidan fácilmente lo que aprenden en clase? ¿Todo el trabajo realizado es inútil? ¿Como aprenden los alumnos? ¿Cómo se aprende? ¿Qué es importante cuando se aprende? ¿Como motivar a los alumnos a aprender?»
Todas estas cuestiones comenzaron de hecho a ser preocupaciones reales de los profesores, influyendo directa e indirectamente en la secuencia de la planificación.

Quien había alcanzado un nivel de comprensión más elevado (caso del profesor A), con una visión de la física más articulada y un sistema conceptual coherente y complejo, intentó profundizar con sus alumnos la visión del mundo que propicia la física:

«Es importante sentir la armonía que existc en la naturaleza y no ponerse en contra de ella. Es necesario que los estudiantes vivencien esas leyes naturales, tal vez ellos solos puedan hacerlo. Presento una cuestión: ¿cuáles son las cualidades del referencial?, ¿es infinito, se extiende por todos lados? Discuto con los alumnos y observo cosas nuevas que despiertan interés.»

De hecho, este profesor fue progresivamente explicitando, para sí y para los otros, la preocupación por la motivación de los estudiantes. Pensaba que, si conseguía mantenerlos interesados durante largos períodos de tiempo discutiendo contenidos relevantes, ei resultado natural sería un aumento de la comprensión. I a metodología sugerida fue la de comenzar discutiendo cuestiones más generales que desarrollasen el espíritu de observación de los estudiantes y permitiesen una participación más amplia, para después introducir "naturalmente» cuestiones más técnicas y ligadas al contenido.

Esta preocupación por la motivación fue compartida rápidamente por los restantes profesores. En una discusión prolongada sobre el tema se lłegó a Ia conclusión de que comprender la física debería ser motivador para los estudiantes. El papel del profesor sería hacerles comprender y convencerlos de que una condición necesaria es estudiar los problemas usando sus propios recursos.

«El aprendizaje exige esfuerzo. Mi nucva actitud en clase exige una actitud diferente de los alumnos, tienen que participar. Planificar no es suficiente para obtener resultados: el alumno debe esforzarse.»

Con este comentario, el profesor $C$ parece haber alcanzado una visión del proceso enseñanza-aprendizaje más realista y articulada. Fue en este punto cuando los profesores comenzaron a abordar en sus planificaciones la cuestión de evaluación más integral, contemplando, además del contenido formal, otros modos de razonamientos más sutiles, actividades ante problemas nuevos, hábitos de trabajo y disposición para aprender. La evaluación pasó a asumir sa papel de guía y control del aprendizaje significativo. Bi profesor C simboliza esta nueva relación con la evaluación de los estudiantes.

«Perdí un día entero en preparar las cuestiones para la evaluación pero quería un instrumento preciso. El resultado fue compensador: conseguí rápidamente detectar quien aprendio y cuáles fueron las dificultades.»

Para este profesor, evaluar dejó de ser sólo atribución de notas; los errores pasaron a ser indicadores importantes en la construcción de conocimiento descable. 
«Con los errores también se aprende, el alumno no debe ser penalizado por eso.s

«Evaluación y motivación están relacionadas. El alumno que recibe una nota $D^{2}$ se desmotiva y desmotiva al profesor."

La motivación dejó de ser para este profesor una palabra general y al mismo tiempo vacía desde el momento que fueron identificados sus aspectos objetivos que caracterizan la disposición e interés para aprender física.

El profesor $F$, al hacer autocrítica de su planificación anterior, comentaba:

«Mi planificación no tenía en cuenta motivar al alumno. El problema es motivar al alumno para que él se pregunte el porqué.»

Su crítica fue más lejos dirigiéndose hacia el proceso de «recuperación» adoptado por las instituciones escolares brasileñas en virtud del cual un estudiante suspenso puede someterse a una evaluación final ${ }^{3}$ :

«Si el alumno no alcanzó los objetivos mínimos propuestos durante el curso tiene derecho a una nueva oportunidad. ¿La nueva oportunidad envuelve nuevas estrategias que permiten el aprendizaje? ¿O la nueva oportunidad es en todo igual a la deI curso?»

El profesor $C$ llegó a la misma conclusión por otro camino. Después de haber descubierto que algunos de sus alumnos realizaban las tareas de casa mecánicamen. te sin preocuparse por comprender, decidió pedir explicaciones individuales sobre las actividades realizadas forzándolos a darse cuenta de lo que hacian. Obtuvo un doble efecto: los estudiantes se esforzaron mucho más en comprender y defender su trabajo y el profesor tuvo mayores oportunidades de entender las dificultades de sus alumnos y percibir sus necesidades diferenciadas. La planifícación de este profesor fue, entonces, más lejos en la preocupación por el aprendizaje de sus alumnos: Ias actividades en clase comenzarían casi siempre con una tarea a ser realizada en pequeños grupos; de esta manera, el profesor, al inspeccionar el desarrollo de los trabajos, podría localizar a los estudiantes con mayores dificultades y dedicarles una atención especial.

En este sentido, el comentario del profesor C es paradigmático, mostrando el inicio de un cambio de perspectiva que se concrete en la atención al aprendizaje efectivo de sus alumnos. Tal «cambio conceptual» en relación con la noción de enseñanza-aprendizaje es el que se espera de un curso de actualización para profesores en activo.

\section{FASES DEL CAMBIO CONCEPTUAL EN LA ACTUALIZACIÓN DE LOS PROFESORES}

En primer lugar podemos afirmar que hubo un cambio significativo. Las ideas de los profesores con relación a la ley de inercia se modificaron abandonando prácticamente los indicios más fuertes de concepciones alternativas. Las ideas subre el proceso enseñanza-aprendizaje fueron reelaboradas asumiendo una visión constructivista del aprendizaje. El cambio más importante fue alcanzado con relación a ta actitudi con los alumnos. Los profesores más sensibles, aunque reconocian, al final del curso que el proceso de aprendizaje dependía fundamen. talmente del esfuerzo y de la dedicación de los alumnos, consideraban que su responsabilidad profesional era estimular efectivamente este esfuerzo, proponer actividades al alcance de sus alumnos y dirigir prioritariamente su proceso de cambio.

Con todo, resulta más importante la posibilidad de caracterizar el proceso de cambio de los profesores mediante etapas sucesivas y probablemente jerárquicas:

a) Organización y perfeccionamiento del propio conocimiento científico. Los problemas han de tratarse cuantitativamente con rigor y exactitud conceptual. Para el profesor, este material es también un bagaje importante que le ayuda en su planificación.

b) Elección de actividades significativas para elaborar la planificación. Los ejemplos presentados y discutidos exhaustivamente en el curso mostraban situaciones en las que se enfrentan concepciones científicas y alternativas. Éstas capacitan al profesor para organizar una secuencia de actividades cuya finalidad es construir concepciones físicas correctas en los estudiantes.

c) Focalización del aprendizaje y su relación con la motivación en clase. La atención del profesor pasa por el proceso de modificación y reclaboración de esas concepciones por medio de una evaluación continua de las dificultades, del progreso de sus alumnos, de adaptación de las actividades a las necesidades de los estudiantes y a los recursos disponibles.

Es posible darse cuenta también que caưa una de esas etapas corresponde a un cambio de perspectiva y a una concepción diferente de sus funciones como profesor. Ello implica una valorización de su papel como orientador del aprendizaje. La perspectiva inicial en relación con la enseñanza era encontrar actividades didácticas ya confeccionadas que pudiesen ser inmediatamente aplicadas en clase. Su función sería la de repasar las actividades interesantes. Cuando se alcanzó una relativa seguridad con el contenido científico y alguna capacidad para actuar como fuente de información y control científico en clase, los profesores dirigieron su atención hacia la planificación con actividades adecuadas para tratar las concepciones de los estudiantes. Tomaron conciencia de su papel de organizadores y planificadores con metas bien definidas y específicas en relación con el contenido científico. Finalmente, en la úitima etapa, los profesores percibieron que su función más significativa era promover, incentivar y sustentar el interés de los alumnos por el aprendizaje, atenuando Ios impedimentos y obstáculos más descorazonadores. 


\section{NUEVA CONCEPCIÓN DE ENSEÑANZA DE LA FISICA Y NUEVA (?) CONCEPCION DE ACTUALIZACION}

Si consideramos que se estableció una nueva concepción de enseñanza, sería interesante relacionarla con el desarrollo del programa de actualización, sus actividades y dinámica de interacción. ¿Qué mecanismos les habrían llevado a una nueva expectativa, aproximándolos más a la de profesional de la educación? ¿Cuáles fueron los elementos del proceso establecidos en el curso de actualización que hicieron posible y estimularon tal cambio? ¿Fue quizás el cambio de orientación desde el inicial «repasador de actividades» hasta «monitor del aprendizaje»?

Resultó interesante y productivo el contacto con los problemas y cuestiones de física extraidos, en gran parte, de las investigaciones ya publicadas. Para los profesores, esta actividad desarrollada en gran parte del curso reveló no sólo que sus conocimientos científicos presentaban lagunas sino que esas lagunas representaban situaciones críticas que debían ser discutidas y comprendidas para la construcción de conocimiento correcto.

La familiaridad de los profesores con las respuestas típicas que encontraban en sus clases y el reconocer en las dificultades de los estudiantes las dudas que ellos mismos tuvieron que vencer, debe haber favorecido un cambio del foco de atención hacia las concepciones de los alumnos.

Hasta ese punto el progreso de vartos profesores fue casi el mismo. Sin embargo, fue más complicado resolver el problema del aprendizaje significativo y duradero de un contenido científico bien establecido.

Fueron resultados concretos de su actuación: la seguridad del profesor en relación con el contenido, la aplicación de su programación en clase, su disposición para enfrentar y discutir las preguntas y errores de sus alumnos. Al parecer son ingredientes esenciales: tener claro y ser conscientes de que las concepciones alternativas están detrás de los errores y poseer un bagaje de problemas y actividades para utilizar en los momentos en que las dificultades son detectadas.

Pasar a dirigir el progreso de los alumnos requería un mayor conocimiento del contenido y, sobre todo, una capacidad de anátisis reflexivo sobre el proceso de aprender y las variables de las que depende éste. El resultado concreto de actuación del profesor en esta situación fue la percepción de la importancia de la evaluación constante con una función directiva más allá de la valoración. Por lo que pudimos constatar durante el curso, sólo los profesores que consiguieron acompañar las actividades de los alumnos de una forma suficientemente flexible para modificarlas cuando las reconocian poco efectivas, fueron también capaces de discriminar jos éxitos parciales de sus alumnos, evaluar su progreso real, asî́ como percibir sus diferencias. Parece ser que este último paso es el efecto natural del hábito de reflexión de los profesores sobre los resultados de su trabajo.

El análisis del progreso de los profesores tienc consecuencias significativas sobre las estrategias de los coordinadores de un curso de actualización. En primer lugar. un curso de actualización necesita tener presente que los profesores modifican sus actuaciones a lo largo del curso, pero lo más importante es que sus intereses y perspectivas también se pueden modificar. Parece una actividad importante de partida el análisis de sus propias actuaciones. Más adelante resulta necesaria la adquisición de alguna autonomía, actividades de enriquecimiento de su fondo de material didáctico y discusiones para analizar tos resultados obtenidos en clase. Consecuentemente el papel de los coordinadores se (lesplaza progresivamente de una situación inicial con actividades de perfeccionamiento científico y didáctico bien definidas hacia una situación final con actividades más flexibles de asesoramiento en la planificación de la unidad didáctica.

Parece también importante prestar atención a las diferencias entre los profesores. Al comienzo del proceso éstas se encuentran principalmente en la comprensión del contenido con una relativa homogeneidad de estilo de planificación didáctica. Al final, las diferencias se encuentran sobre todo en la visión del papel de las actividades en el proceso de orientación del aprendizaje de los estudiantes y la función de la evaluación an el control de ese proceso. Ello significa que precisa adaptar también la flexibilidad de los coordinadores a las distintas exigencias y perspectivas de varios profesores al mismo tiempo.

Finalmente, una última consideración en relación con las fases de evolución de los profesores. Si fuera verdad que esas fases son jerárquicas y hubiera que recorrerlas en un orden determinado, parecería interesante que los profesores fueran informados sobre este camino. De csta forma, ellos mismos podrían guiar su proceso e incentivar su progreso. De cualquier forma, parece más motivador saber que existen formas más refinadas de planificación escolar, y que el principal indicador de progreso y éxito será que estas formas sean incorporadas de manera natural. 


\section{AGRADECIMIENTO}

Agradecemos al Dr. José María de Posada la fiel y bien cuidada traducción que realizó.

\section{NOTAS}

* Con la financiación parcial del CAPES y del CNPq.

- N.T. El segundo grado comprende tres cursos y está destinado a los alumnos entre 15 y 18 años de edad. Las materias de física y químicá están separadas, los profesores que las imparten pertenecen a departamentos diferentes.

2 N.T. Calificación equivalente a suspenso. Los códigos utilizados varían mucho de una institución a otra.

3 N.T. En el sistema escolar brasileño no existe recuperación después de las vacaciones estivales; ésta se realizâ, normalmente, dos semanas después de terminadas las clases oficiales.

atualização. Revista Braśileira de Ensino de Física, Vol. 14(1). pp. 39-42.

PACCA, J.L.A. y VILLANI, A. (1991). Difficulties of Teachers in Teaching the Law of the Inertia. Proceedings International Conference GIREP: Teaching about Reference Frames: from Copernicus to Einstein, Torun, pp. 387-39I.

PACCA, J.L.A. y VILLANI, A. (1992). Estratégias de ensino e mudança conceitual na atualizaçào de professores. Revista Brasileira de Ensino de Física, Vol. I4(4), pp. 222-228.

P.S.S.C. (1960). Physics. Physical Science Study Committee. Boston: D.C. Heath and Company.

TOBIN, K. y ESPINET, M. (1989). Impediments to change: Applications of coaching in high-school science teaching. Journal of Research in Science Teaching, Vol. 26(1), pp. $105-120$.

VILLANI, A. (1991). Planejamento escolar: um instrumento de atualização dos professores de ciências. Revista de Ensino de Fisica, Vol. 13, pp. 162-177.

VILLANI, A. y PACCA, J.L.A. (1991). Difficulties for Comprehension of the Law of the Inertia. Proceedings International Conference GIREP: Teaching about Reference Frames: from Copernicus to Einstein, Torun, pp. 382-386.

VILLANI, A. y PACCA, J.L.A. (1992). Teoria e Prática Didática na Atualização de Professores de Física. Revista Brasileira de Ensino de Física, Vol. 14(2), pp. 113-119.

ZALAMEA, E. y PARIS, R. (1989). ¿Saben los maestros la física que enseñan? Enseñanza de las Ciencias, Vol. 7(3), pp. 251-256.

[Artículo recibido en enero de 1994 y aceptado en septiembre de 1995.] 
